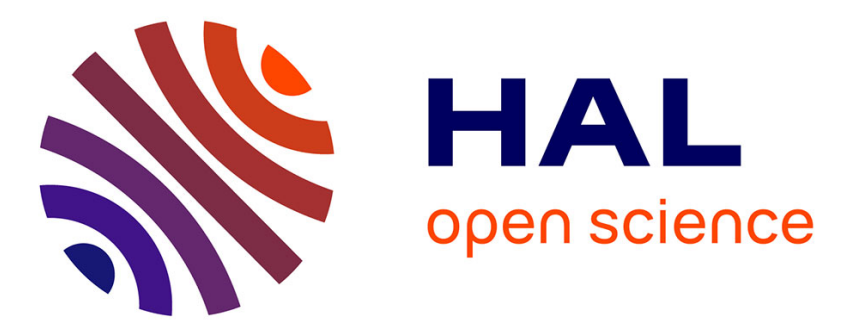

\title{
Les mécanismes de l'oxydation interne dans les alliages à base d'argent
}

\author{
L. Charrin, A. Combe, A. Charai, J. Cabane
}

\section{To cite this version:}

L. Charrin, A. Combe, A. Charai, J. Cabane. Les mécanismes de l'oxydation interne dans les alliages à base d'argent. Journal de Physique IV Proceedings, 1994, 04 (C3), pp.C3-127-C3-132. 10.1051/jp4:1994318 . jpa-00252515

\section{HAL Id: jpa-00252515 https://hal.science/jpa-00252515}

Submitted on 1 Jan 1994

HAL is a multi-disciplinary open access archive for the deposit and dissemination of scientific research documents, whether they are published or not. The documents may come from teaching and research institutions in France or abroad, or from public or private research centers.
L'archive ouverte pluridisciplinaire HAL, est destinée au dépôt et à la diffusion de documents scientifiques de niveau recherche, publiés ou non, émanant des établissements d'enseignement et de recherche français ou étrangers, des laboratoires publics ou privés. 


\title{
Les mécanismes de l'oxydation interne dans les alliages à base d'argent
}

\author{
L. CHARRIN, A. COMBE, A. CHARAI et J. CABANE
}

Laboratoire de Métallurgie, EDIFIS, associé au CNRS, Faculté des Sciences et Techniques de Saint Jérôme, Case 511, 13397 Marseille cedex 20, France

\begin{abstract}
Internal oxidation mechanism in silver alloys was studied by residual resistivity, gravimetric and calorimetric measurements, X-Ray diffraction and by transmission electron microscopy. It has been established in $\mathrm{Ag}-\mathrm{Mg}$ alloys that the oxygen fixation process involves three steps : firstly, substoichiometric species are initially formed; secondly these elementary species evolve toward irregular clusters including an important excess of oxygen; thirdly, a part of this excess oxygen is released and clusters evolve toward a more compact structure. The mechanism of internal oxidation in $\mathrm{Ag}-\mathrm{Mg}$ alloys can be generalized to the other studied alloys
\end{abstract} such as $\mathrm{Ag}-\mathrm{Cu}, \mathrm{Ag}-\mathrm{Zn}$ and $\mathrm{Ag}-\mathrm{Sn}$.

L'oxydation interne est une transformation avec diffusion qui résulte des interactions entre l'oxygène dissous dans un métal et un élément d'addition. En choisissant bien les conditions de la réaction, nous avons montré qu'il était possible d'étudier le phénomène dans son ensemble, depuis le domaine de préprécipitation au cours duquel les produits de l'oxydation sont en solution solide jusqu'à celui de l'apparition de précipités cohérents.

Les premières étapes avant l'apparition de l'oxyde s'accompagnent de phénomènes complexes comme par exemple:

- un accroissement de la résistivité résiduelle(1-3).

- une fixation d'un excès d'oxygène(4-8).

- une dilatation de la maille(9-11).

\section{1- ANOMALIE DE LA RESISTIVITE}

En principe, la formation de la phase oxyde devrait se traduire par une diminution de la résistivité (12-13). Or, au cours des premiers stades de l'oxydation interne, on a mis en évidence (Fig.1) que la résistivité passait par un maximum et diminuait ensuite, et ceci quelle que soit la température d'oxydation et la nature du soluté: étain, zinc, magnésium et cuivre.

On constate que la résistivité mesurée expérimentalement atteint un maximum qui est, en général, plus élevé que la valeur théorique obtenue pour un alliage dans lequel les atomes de soluté seraient en solution solide. Par exemple, un alliage $\mathrm{Ag}-\mathrm{Mg}(0,44 \mathrm{at} \%)$ oxydé à $395^{\circ} \mathrm{C}$ a une résistivité maximale de 3,13 $\mu \Omega \mathrm{cm} /$ at\% alors que la valeur théorique ne serait que de $2,62 \mu \Omega \mathrm{cm} / \mathrm{at} \%$. Cette 
anomalie de résistivité s'interprète par une oxydation sous forme de très petits amas constitués d'atomes d'oxygène en insertion et d'atomes de soluté en substitution. Ces amas ont une résistivité plus élevée que celle des atomes d'oxygène et de soluté distribués au hasard.

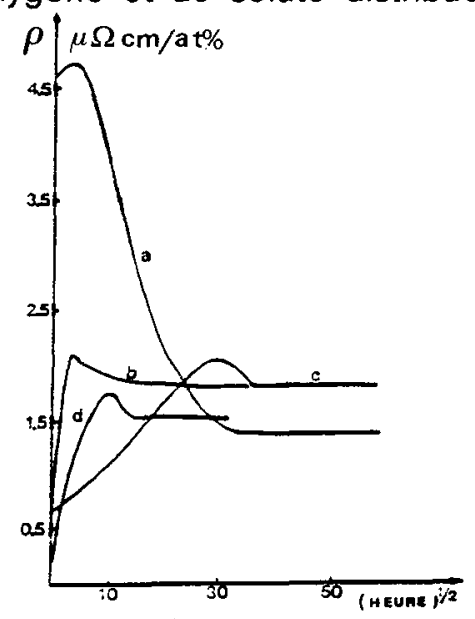

FIG.1 - Variation de la résistivité résiduelle en fonction de $t^{1 / 2}$ pour les alliages d'épaisseur $1 \mathrm{~mm}$ :
(a) $\mathrm{Ag}-\mathrm{Sn}(0,19 \mathrm{at} \%)-400^{\circ} \mathrm{C}-\mathrm{pO}_{2}=1 / 5 \mathrm{~atm}$
(b) $\mathrm{Ag}-\mathrm{Mg}(0,15$ at\% $)-530^{\circ} \mathrm{C}-\mathrm{pO}_{2}=1 / 5 \mathrm{~atm}$
(c) Ag-Zn $(0,22$ at $\%)-400^{\circ} \mathrm{C}-\mathrm{pO}_{2}=1 / 5 \mathrm{~atm}$
(d) $\mathrm{Ag}-\mathrm{Cu}(0,018 \mathrm{at} \%)-300^{\circ} \mathrm{C}-\mathrm{pO}_{2}=1 \mathrm{~atm}$

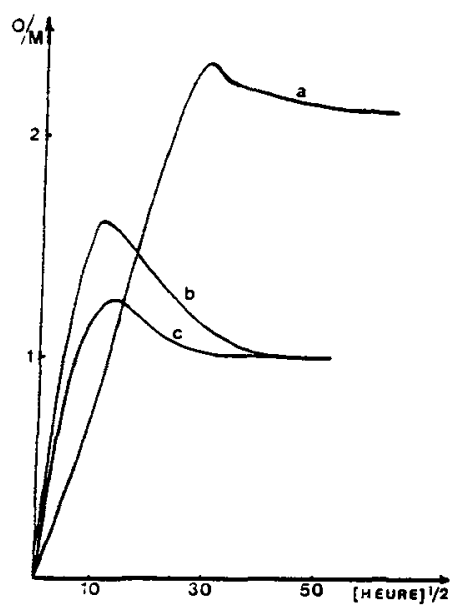

FIG. 2 : Variation du rapport $O / M$ en fonction de $t^{1 / 2}$ pour 3 alliages d'épaisseur $1 \mathrm{~mm}$ :
(a) $\mathrm{Ag}-\mathrm{Sn}(0,19 \mathrm{at} \%)-400^{\circ} \mathrm{C}-\mathrm{pO}_{2}=1 / 5 \mathrm{~atm}$
(b) $\mathrm{Ag}-\mathrm{Cu}(0,072$ at $\%)-300^{\circ} \mathrm{C}-\mathrm{pO}_{2}=1 \mathrm{~atm}$
(c) $\mathrm{Ag}-\mathrm{Mg}(0,15 \mathrm{at} \%)-530^{\circ} \mathrm{C}-\mathrm{pO}_{2}=1 / 5 \mathrm{~atm}$

\section{2 - FIXATION D'UN EXCES D'OXYGENE}

On a étudié la variation du rapport oxygène/métal, soit $O / M$, en fonction du temps $\left(t^{1 / 2}\right)$ dans le domaine des basses températures $\left(<600^{\circ} \mathrm{C}\right)$, pour 3 alliages $\mathrm{Ag}-\mathrm{Mg}, \mathrm{Ag}-\mathrm{Sn}$ et $\mathrm{Ag}-\mathrm{Cu}$ (14).

On peut voir que, quel que soit le soluté, le rapport $\mathrm{O} / \mathrm{M}$ augmente, passe par un maximum et diminue ensuite jusqu'à la valeur correspondant à l'oxyde stoechiométrique (soit 2 pour l'alliage $\mathrm{Ag}-\mathrm{Sn}$ et 1 pour les alliages $\mathrm{Ag}-\mathrm{Cu}$ et $\mathrm{Ag}-\mathrm{Mg}$ ).

La FIG.2 met en évidence:

- la fixation d'un excès d'oxygène par rapport à l'oxyde stoechiométrique.

- une élimination d'une partie de cet oxygène en excès.

\section{3 - ACCROISSEMENT DU PARAMETRE CRISTALLIN}

La diffraction des $\mathrm{R}-\mathrm{X}$ a permis de mesurer, à la surface des échantillons, les variations du paramètre cristallin en fonction du temps $\left(\mathrm{t}^{1 / 2}\right)$ pour deux alliages $\mathrm{Ag}-\mathrm{Mg}(1 \mathrm{at} \%)$ et $\mathrm{Ag}-\mathrm{Cu}(0,2 \mathrm{at} \%)$.

On constate que, quel que soit le soluté, le paramètre augmente, passe par un maximum et décroît jusqu'à une valeur finale qui est plus élevée que la valeur 
initiale dans le système $\mathrm{Ag}-\mathrm{Mg}$, et voisine de la valeur initiale pour le système $\mathrm{Ag}-\mathrm{Cu}$.

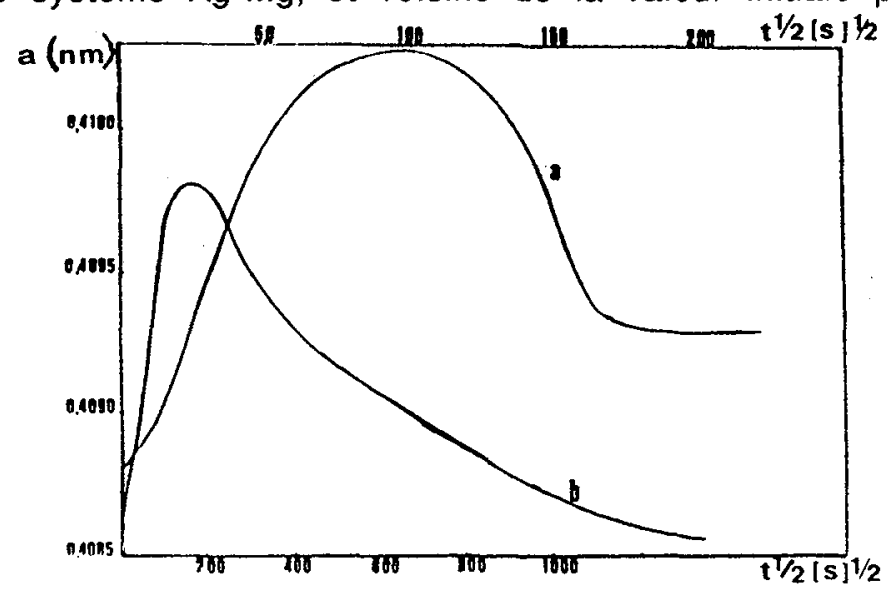

FIG.3 - Variation de la maille cristalline en fonction de $t^{1 / 2}$ pour 2 alliages :

(a) $\mathrm{Ag}-\mathrm{Mg}(1 \mathrm{at} \%)-300^{\circ} \mathrm{C}-\mathrm{PO}_{2}=1 / 5 \mathrm{~atm}$

(b) $\mathrm{Ag}-\mathrm{Cu}(0,2 \mathrm{at} \%)-300^{\circ} \mathrm{C}-\mathrm{PO}_{2}=1 / 5 \mathrm{~atm}$

Ces anomalies observées pour tous les systèmes, s'expliquent par une oxydation sous forme d'amas dans lesquels les atomes de soluté et d'oxygène sont en solution. Une fois formés ces amas n'évoluent pas de manière significative; cet effet est particulièrement marqué dans un système comme $\mathrm{Ag}-\mathrm{Mg}$ dans lequel le soluté a une forte affinité pour l'oxygène.

\section{ETUDE DU SYSTEME Ag-Mg}

\section{- OXYDATION A BASSE TEMPERATURE $\left(280-500^{\circ} \mathrm{C}\right)$}

L'oxydation interne dans des alliages $\mathrm{Ag}-\mathrm{Mg}$, de concentration 0,12 à 1,55at\%,a été effectuée sur des plaquettes polycristallines d'épaisseur variable de façon à mettre en évidence une éventuelle distribution en taille des amas en fonction de la distance à partir de la surface. Cette étude est réalisée par résistivité résiduelle, gravimétrie, calorimétrie (15-16), diffraction des $R-X$ et par microscopie électronique.

Nous avons porté sur la Fig.4 les variations du rapport $\mathrm{O} / \mathrm{Mg}$, la résistivité résiduelle $\rho$ et la quantité de chaleur $Q / S$ en fonction de $t^{1 / 2}$ pour un alliage $\mathrm{Ag}-\mathrm{Mg}$ (1at\%) oxydé à $420^{\circ} \mathrm{C}$ sous une pression de $1 \mathrm{~atm}$.

Toutes ces courbes montrent que la fixation de l'oxygène s'effectue en trois périodes:

1 - La première correspond à la fixation immédiate de l'oxygène quelle que soit la température avec une cinétique relativement lente ,un faible dégagement de chaleur et une dilatation non observable (17). 


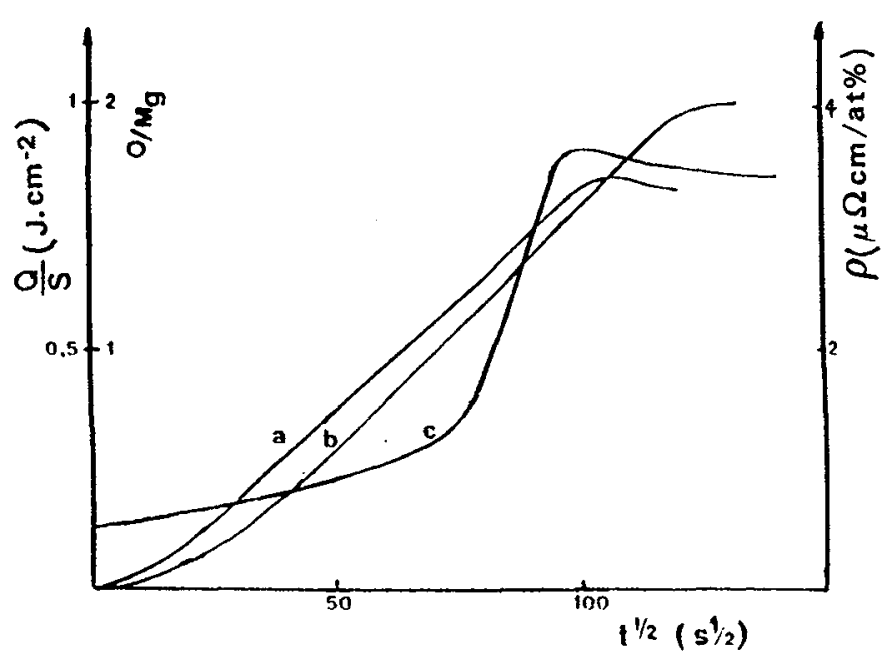

FIG 4 - Variation du rapport $\mathrm{O} / \mathrm{Mg}$ (a), la quantité de chaleur $\mathrm{Q} / \mathrm{S}\left(\mathrm{J} . \mathrm{cm}^{-2}\right)$ (b)et de la résistivité résiduelle $\rho(\mu \Omega \mathrm{cm} / \mathrm{at} \%)$ (c) en fonction de $\mathrm{t}^{1 / 2}$ pour un alliage $\mathrm{Ag}$ $\mathrm{Mg}$ (1 at\%) d'épaisseur $9.10^{-3} \mathrm{~cm}-400^{\circ} \mathrm{C}-\mathrm{PO}_{2}=1 \mathrm{~atm}$.

2 - La seconde correspond à une accélération de la fixation de l'oxygène avec un accroissement important de la résistivité et du paramètre cristallin et un fort dégagement de chaleur.

3 - La troisième période correspond à l'élimination d'une partie de l'oxygène en excès ; elle s'accompagne d'une diminution de la résistivité et du paramètre cristallin et d'un dégagement de chaleur.

Les caractéristiques de ces amas formés à basse température ne semblent pas dépendre de la distance à partir de la surface lorsque celle-ci est supérieure à une vingtaine de microns comme le montrent les mesures de paramètre (17) effectuées à differentes distances de la surface ainsi que les quantités de chaleur mesurées en fonction de l'epaisseur des échantillons (14)..

\section{- EVOLUTION AVEC LA TEMPERATURE APRES OXYDATION.}

Lorsqu'on effectue des traitements thermiques successifs sur un alliage $\mathrm{Ag}-\mathrm{Mg}$ $(0,89$ at $\%)$ oxydé à basse température $\left(530^{\circ} \mathrm{C}\right)$, on constate que l'évolution du système $\mathrm{Ag}-\mathrm{Mg}$ est très lente (3). La résistivité résiduelle reste très élevée; il faut une température de $700^{\circ} \mathrm{C}$ pour noter une diminution notable de la résistivité. L'état d'équilibre est atteint difficilement même à haute température.

\section{- OXYDATION A HAUTE TEMPERATURE.}

Lorsqu'un alliage $\mathrm{Ag}-\mathrm{Mg}$ est oxydé directement à haute température $\left(800^{\circ} \mathrm{C}\right)$, l'évolution de la résistivité résiduelle est comparable à celle observée au cours d'une oxydation effectuée à $530^{\circ} \mathrm{C}$ suivie d'une augmentation progressive de la température jusqu'à $800^{\circ} \mathrm{C}(2)$.

Après un recuit oxydant à $850^{\circ} \mathrm{C}$ de 8 jours, nous avons observé en MET des 
précipités cohérents de 5 à $6 \mathrm{~nm}$ qui correspondent à l'oxyde stoechiométrique $\mathrm{MgO}$ (18-19) Ces résultats montrent que des températures élevées et des traitements thermiques de longue durée sont nécessaires pour observer des précipités cohérents.

De l'ensemble de ces résultats, on peut proposer un mécanisme de formation de l'oxyde.

\section{MECANISME DE FORMATION DE L'OXYDE.}

- La fixation de l'oxygène à partir de la surface est immédiate: il n'y a pas de période d'incubation. Les produits de l'oxydation sont constitués par des associations entre les atomes métalliques en substitution et les atomes d'oxygène en insertion.On les désignera par "espèces élémentaires ". Elles se forment avec un dégagement de chaleur appréciable ,dilatent peu le réseau de l'argent et fixent une quantité d'oxygène inférieure à la valeur correspondant à la stoechiométrie. Ces premières espèces sont globalement sous stoechiométriques. La plus simple est l'espèce $(\mathrm{MgO})^{*} . I l$ est aussi possible d'envisager des espèces telles que $\left(\mathrm{Mg}_{2} \mathrm{O}\right)^{*},\left(\mathrm{Mg}_{3} \mathrm{O}\right)^{*}$ par diffusion à courte distance du magnésium vers l'espèce préalablement formée $(\mathrm{MgO})^{*}$.

- Le système évolue ensuite par coalescence de ces espèces élémentaires. On obtient alors des "amas". Au cours de cette évolution, ils fixent une quantité d'oxygène supérieure à celle correspondant à la stoechiométrie de l'oxyde $\mathrm{MgO}$. Leur structure imparfaite et peu compacte entraîne des déformations locales très importantes qui sont dues essentiellement à la position interstitielle de l'oxygène dans ces amas. Ces déformations se traduisent par un accroissement considérable du paramètre cristallin, et par l'existence d'un grand nombre de dislocations observées en Microscopie Electronique à Transmission. Ces amas sont de très petite taille. En effet, l'accroissement important de la résistivité met l'accent sur l'existence de rassemblements de petite taille dans un état de grande dispersion.

- Ces amas de structure peu compacte et créant des déformations importantes se réarrangent de manière à devenir plus compacts et plus stables. $\mathrm{Au}$ cours de cette transformation, il y a une élimination partielle de l'oxygène en excès, une disparition progressive des dislocations (18), une diminution de la résistivité et du paramètre cristallin. On obtient des amas cohérents visibles en M.E.T .Ces amas $\mathrm{Mg}_{n} \mathrm{O}_{m}$ formés évoluent difficilement ,vu la faible concentration en espèces mobiles $\left(\mathrm{Mg}^{\mathrm{MgO}} \mathrm{MgO}^{*}\right.$ qui pourraient permettre leur grossissement. Seule l'augmentation de la température peut provoquer une évolution de ces amas vers l'oxyde stoechiométrique. Les amas ayant atteint une certaine taille et une certaine compacité évoluent vers l'oxyde stoechiométrique $\mathrm{MgO}$ suivant la réaction:

( $\mathrm{MgO}$ )amas -------> $\mathrm{MgO}$ oxyde stoechiométrique 
Cette réaction s'effectue à haute température par un phénomène de "mûrissement d'Oswald."

\section{CONCLUSION}

L'oxydation interne dans des alliages à base d'argent a permis de suivre un phénomène de précipitation dans son ensemble, depuis le domaine de préprécipitation au cours duquel les produits de l'oxydation sont en solution jusqu'à l'apparition de précipités cohérents. Les résultats obtenus peuvent se généraliser pour tous les autres systèmes étudiés $\mathrm{Ag}-\mathrm{Cu}, \mathrm{Ag}-\mathrm{Zn}$ et $\mathrm{Ag}-\mathrm{Sn}$.

\section{REFERENCES}

/1/ KLEIN M.J. et HUGGINS R.A., Trans. Am. Soc. Met., 55 (1962), 14

121 COMBE A., BERNARDINI J. et CABANE J., Mem. Scient. Rev Metal.,(1976), 149

/ 3 / COMBE A., CHARRIN L., MOYA G. et CABANE J., Acta Met., 31, N7, (1983), 1019

/ 4 / GURUSWAMY S., PARK S.M., HIRTH J.P. et RAPP R.A., Oxid. Met, 26, (1986), 77

$15 /$ PETHE L.D., MATHUR H.B. et BISWAS A.B;, Can. J. Chem., 46, (1968), 1187

$16 /$ COMBE A. et CABANE J., Oxid. Met., 21, (1984), 21

$17 /$ CHARRIN L., COMBE A. et CABANE J., Oxid. Met., 37, (1992), 65

/ 8 / SEMEGA B.M., CHARRIN L., COMBE A. et ARIDE J., Phil. Mag. A, 66, (1992), 1139

/ 9 / KRAWITZ A. et SINCLAIR R., Phil. Mag., 31, (1975), 697

/10/ HIRSCHHORN - Met. Sci. J., 1, (1967), 91

111 / CHARRIN L., COMBE A., CABANE F. et CABANE J., Oxid. Met.,40,(1993),483

112 / LUIGGI N., SIMON J.P. et GUYOT P., J. Phys. F, 10, (1980), 865

/1 3/ LUIGGI N., SIMON J.P; et GUYOT P., Acta Metall., 28, (1980), 1155

$114 /$ COMBE A. - Thèse, Marseille 1982

$115 /$ CHARRIN L., COMBE A. et MOYA G., J. Therm. Anal., 14, (1978), 89

/16/CHARRIN L. - Thèse, Marseille 1987

117 / CHARRIN L,COMBE A,CHARAI A,CABANE F,CABANE J,(ce colloque)

118 / CHARAI A., COMBE A., BOULESTEIX C. et CABANE J., Scripta. Metall., 17, (1983), 833

/19/ CHARAI A., NIHOUL G. - Phil. Mag. A, 58, (1988), 57 